\title{
MR molecular imaging of tumours using ferritin heavy chain reporter gene expression mediated by the hTERT promoter
}

\author{
Yan Yang ${ }^{1,2}$ • Ming-fu Gong ${ }^{1}$ • Hua Yang ${ }^{1}$ - Song Zhang ${ }^{1}$ - Guang-xian Wang ${ }^{1}$. \\ Tong-sheng $\mathrm{Su}^{1} \cdot \mathrm{Li}^{1} \mathrm{Wen}^{1} \cdot$ Dong Zhang ${ }^{1}$
}

Received: 2 June 2015 /Revised: 6 December 2015 / Accepted: 28 January 2016 /Published online: 9 March 2016

(C) The Author(s) 2016. This article is published with open access at Springerlink.com

\begin{abstract}
Objectives Using the human telomerase reverse transcriptase (hTERT) promoter and the modified ferritin heavy chain (Fth) reporter gene, reporter gene expression for MRI was examined in telomerase positive and negative tumour cells and xenografts.

Methods Activity of the reporter gene expression vector Lenti-hTERT-Fth1-3FLAG-Puro was compared to constitutive CMV-driven expression and to the untransfected parental control in five tumour cell lines: A549, SKOV3, 293T, U2OS and HPDLF. In vitro, transfected cells were evaluated for FLAG-tagged protein expression, iron accumulation and transverse relaxation. In vivo, tumours transduced by lentiviral vector injection were imaged using $\mathrm{T} 2 * \mathrm{WI}$. Changes in tumour signal intensity were validated by histology.

Results Only telomerase positive tumour cells expressed FLAG-tagged Fth and displayed an increase in R2* above the parental control, with a corresponding change in $\mathrm{T} 2 * \mathrm{WI}$. In addition, only telomerase positive tumours, transduced by injection of the reporter gene expression construct, exhibited a change in signal intensity on T2*WI. Tumour histology verified the expression of FLAG-tagged Fth and iron accumulation in telomerase positive tissue.
\end{abstract}

Li Wen

cqzdw1@163.com

Dong Zhang

hszhangd@163.com

1 Department of Radiology, XinQiao Hospital, Third Military Medical University, ChongQing 400037, People's Republic of China

2 Department of Radiology, The First Affiliated Hospital of ChengDu Medical College, ChengDu 610500, People's Republic of China
Conclusion Reporter gene expression for MRI, using the Fth reporter and the hTERT promoter, may be a useful strategy for the non-invasive diagnosis of many types of cancer.

Key points

- Modified heavy chain of ferritin can serve as an MR reporter gene

- hTERT promoter can direct the expression of reporter gene in cancer cells

- MR reporter imaging mediated by hTERT promoter can be used for cancer diagnosis

Keywords Magnetic resonance imaging $\cdot$ Neoplasm . hTERT $\cdot$ Reporter gene expression $\cdot$ Ferritin heavy chain

$\begin{array}{ll}\text { Abbreviations } \\ \text { CMV } & \text { Cytomegalovirus } \\ \text { ELISA } & \text { Enzyme-linked immunosorbent assay } \\ \text { FAC } & \text { Ferric ammonium citrate } \\ \text { FSE } & \text { Fast spin echo } \\ \text { Fth } & \text { Ferritin heavy chain } \\ \text { hTERT } & \text { Human telomerase reverse transcriptase } \\ \text { GRE } & \text { Gradient recalled echo } \\ \text { MRI } & \text { Magnetic resonance imaging } \\ \text { TE } & \text { Time to echo } \\ \text { TR } & \text { Time to repetition } \\ \text { T2*WI } & \text { T2*-weighted imaging } \\ \text { T2WI } & \text { T2-weighted imaging }\end{array}$

\section{Introduction}

Telomerase is a ribonucleoprotein enzyme that controls the elongation of telomeres in mammalian cells [1]. It is usually repressed in most somatic cells and reactivated in 
approximately $85 \%$ of human cancer cells, which maintain telomerase activity to sustain proliferation [2]. The selective reactivation of telomerase in cancer cells offers an attractive target for cancer diagnosis. Human telomerase reverse transcriptase (hTERT) is the catalytic subunit of telomerase, and a strong correlation is observed between telomerase activity and hTERT mRNA expression in a variety of cancers [3]. Many researchers designed reporter gene imaging formulations in which promoter fragments of hTERT were used to direct the expression of reporter genes [4]. These encoded for proteins that change MRI contrast, bind radiolabelled probes or emit light $[5,6]$. By introducing the gene expression system into cells using molecular techniques, the reporter gene of choice was (over)expressed only in telomerase-positive tumour cells as demonstrated in vitro and in vivo by position emission tomography (PET) or charge-coupled device (CCD) camera using reporter genes such as the $\mathrm{Na} / \mathrm{I}$ symporter (NIS) [7] or the green fluorescence protein (GFP) [8].

MRI has been widely used in the field of modern medical diagnosis and treatment. Compared with optical imaging and nuclear medicine modalities, MRI has the advantages of multi-parameter imaging, higher spatial and temporal resolution, and lack of ionizing radiation. The primary requirement for an MR reporter gene is to provide contrast that can be distinguished from the non-transgenic cells [9]. Ferritin is an iron storage protein, which can accumulate significant amounts of hydrous ferric oxide iron from the intracellular labile iron pool, and then store it as ferrihydrite [10]. The expression of ferritin causes the transverse relaxation rate of transfected cells to increase significantly and generate strong hypointensity on $\mathrm{T} 2 / \mathrm{T} 2 *$-weighted images [10].

In the present study, we hypothesized that telomerasepositive tumours and cells would be visualized by MR imaging using the hTERT promoter and the modified ferritin heavy chain (Fth) reporter gene. To test our hypothesis, we generated recombinant lentivirus Lenti-hTERT-Fth1-3FLAG-Puro in which the hTERT promoter was used to direct the expression of the modified Fth reporter gene, and then the recombinant vectors were transduced into cells in vitro and injected into tumour models in nude mice. Our aim was to assess the potential application of MR reporter imaging, mediated by the hTERT promoter, to cancer diagnosis.

\section{Materials and methods}

\section{Construction and production of lentiviral vectors}

The ferritin heavy chain (Fth1) gene was synthesized by Shanghai Generay Biotech Co., Ltd according to the Fth1 gene sequence in GenBank (NM_010239) with the exclusion of regulatory iron response elements, and then tagged with three consecutive FLAG sequence (3FLAG). The construct was subcloned into the NheI and BamHI restriction sites in the lentiviral vector pCDH-CMV-MCS-Puro to form the pCDH-CMV-Fth1-3FLAG-Puro vector. The pCDH-CMVFth1-3FLAG-Puro vector was linearized by restriction digest with SpeI and $X b a \mathrm{I}$, and purified to remove the CMV promoter fragment. Polymerase chain reaction (PCR) was used to amplify the hTERT promoter from plasmid pLenti-hTERT-EGFP, a gift from Mr Li (Department of Pathophysiology, Third Military Medical University, Chongqing, China), using forward primer 5' - T T T TAT C GATA C TA G T C A C A GA C GCCCA GGACCGCGCTTC-3' and reverse primer 5' ATTCGCTA GCTCTAGACCACGTGCGCCCAC GTGCGCCCAC-3' containing SpeI and $X b a \mathrm{I}$ restriction sites, respectively. After restrictive digestion with $S p e I$ and $X b a \mathrm{I}$, the hTERT promoter fragment was successfully inserted into the linearized plasmid to form the pCDH-hTERT-Fth1-3FLAGPuro vector.

Lentiviral vectors were produced by cotransfecting 293T cells with three plasmids (the reconstructed plasmids pCDHhTERT-Fth1-3FLAG-Puro or pCDH-CMV-Fth1-3FLAGPuro, the packaging plasmids $\mathrm{pCD} / \mathrm{NL}-\mathrm{BH} * \mathrm{DDD}$ and pLTR-G) at a ratio of 3:2:1. Forty-eight hours after transfection, the viruses were collected and concentrated. The constructed lentiviral vectors were named Lenti-hTERT-Fth13FLAG-Puro and Lenti-CMV-Fth1-3FLAG-Puro.

\section{Culture of cells}

Five human cell lines were chosen for this study. Lung carcinoma A549, ovarian carcinoma SKOV3, embryonic kidney 293T, osteosarcoma U2OS and primary periodontal ligament fibroblast (HPDLF) cells were all obtained from the central laboratory of Xin Qiao Hospital (Chongqing, China). A549, SKOV3, 293T and HPDLF cells were maintained in DMEM medium (Gibco), and U2OS cells were cultured in McCoy's $5 \mathrm{~A}$ medium (Gibco). All media were supplemented with $10 \%$ fetal bovine serum (Gibco) and $100 \mathrm{U} / \mathrm{ml}$ of penicillin-streptomycin (Gibco). Immunofluorescence staining and western blotting were performed as described below to determine the presence of hTERT protein in all cell lines.

\section{Lentivirus transfection}

Cells were incubated with Lenti-hTERT-Fth1-3FLAG-Puro and Lenti-CMV-Fth1-3FLAG-Puro in the presence of polybrene (Sigma, 5-8 $\mu \mathrm{g} / \mathrm{ml}$ ) for $24 \mathrm{~h}$ [11]. The expression of FLAG-tagged Fth in all cells was analysed by immunofluorescence staining and an enzyme-linked immunosorbent assay (ELISA) as described below. Iron accumulation was determined by Prussian blue iron staining and by using a total iron assay kit as described below. 


\section{Western blot analysis}

Cells $\left(2 \times 10^{6}\right)$ were lysed on ice in $200 \mu$ RIPA buffer with protease inhibitor (Sigma). The concentration of the collected cellular protein was evaluated by the bicinchoninic acid (BCA) assay according to the manufacturer's instructions (Pierce Biotechnology) [12], and ca. $15 \mu \mathrm{g}$ of the protein samples was loaded onto $10 \%$ gels for SDS-PAGE. The proteins were then transferred onto PVDF membranes for blotting. The membranes were blocked in $5 \%$ bovine serum albumin for $2 \mathrm{~h}$ and then incubated overnight at $4{ }^{\circ} \mathrm{C}$ with rabbit anti-TERT monoclonal antibody (1:1000, Abcam) or mouse anti- $\beta$-actin monoclonal antibody (1:1000, Abcam). After thoroughly washing with Tris-buffered saline with $0.1 \%$ Tween 20 ( $0.1 \%$ TBST), the membranes were incubated with horseradish peroxidase-conjugated goat anti-rabbit or anti-mouse IgG (1:1000, Santa Cruz) for $1 \mathrm{~h}$. The protein bands were visualized by enhanced chemiluminescent (ECL) agents (Pierce Biotechnology) following the manufacturer's protocol.

\section{Immunofluorescence staining}

Cells were cultured in confocal petri dishes (NEST Biotechnology) and rinsed with phosphate buffered saline (PBS) $\mathrm{pH} 7.4$ before examination. Following fixation in $4 \%$ paraformaldehyde (PFA) for $10 \mathrm{~min}$, the cells were blocked with $5 \%$ normal goat serum in PBS at $37{ }^{\circ} \mathrm{C}$ for $30 \mathrm{~min}$. To examine hTERT protein expression, the cells were incubated with rabbit anti-hTERT monoclonal antibody (1:500, Abcam) overnight at $4{ }^{\circ} \mathrm{C}$ and then visualized with Alexa Flour 488conjugated goat anti-rabbit antibody (1:1000, Beyotime). To examine FLAG protein expression, the cells were incubated with mouse anti-FLAG monoclonal antibody (1:1000, Sigma) overnight at $4{ }^{\circ} \mathrm{C}$ and then visualized with Alexa Flour 555conjugated goat anti-mouse antibody (1:1000, Beyotime). The cell nuclei were counterstained with DAPI (1:1000, Beyotime).

\section{Enzyme-linked immunosorbent assay}

Extracts of $10^{6}$ cells were collected, and the BCA assay was used to determine the total protein concentration of each sample. The total ferritin heavy chain (Fth) content in each cell sample was analysed using an ELISA kit (Uscn Life Science Inc, Wuhan, China) according to the manufacturer's instructions. The concentration of Fth in the samples was determined by comparing the O.D. of the samples to the standard curve. The results are expressed as nanograms of Fth per milligram of total protein. Values are the means $\pm \mathrm{SD}$ of three independent experiments.

\section{Assessment of iron accumulation}

Cells were cultured in medium supplemented with ferric ammonium citrate (FAC, $1 \mathrm{mM}$, Sigma) for $48 \mathrm{~h}$ and then washed thoroughly with PBS to remove any free iron. For Prussian blue staining, cells were fixed in $4 \%$ PFA for 10 min and then incubated in Prussian blue working solution, which was prepared by mixing equal parts of $1 \%$ potassium ferric ferrocyanide solution and $5 \%$ hydrochloric acid solution for $30 \mathrm{~min}$ [12]. Cells were counterstained with nuclear fast red (Beyotime) for $5 \mathrm{~min}$. The iron content of all cells was further determined by using an iron assay kit according to the manufacturer's instructions (Sigma). Briefly, $2 \times 10^{6}$ cells were homogenized in cold iron assay buffer, and the cell extracts were collected to determine their iron contents. Absorbance was immediately measured at $593 \mathrm{~nm}$. The iron content for each sample was determined by dividing the iron content by the number of cells (pg/cell). Three sets of samples were measured.

\section{Tumour models}

Twelve BALB/c nude mice (male, 4 weeks old, and 16-18 g) of specific pathogen-free grade were purchased from the Animal Department, Third Military Medical University. We performed experiments in accordance with the local ethics committee of the Third Military Medical University. The mice were treated with an intraperitoneal injection of $400 \mu \mathrm{l}$ iron dextran $(50 \mathrm{mg} / 2 \mathrm{ml}$, Zhejiang Ruian Pharmaceutical Factory, China) every 3 days for a total of six injections. After the fourth iron injection, the bilateral hind flanks of the mice were subcutaneously inoculated with A549 or U2OS cells $\left(10^{6}\right.$ cells/position) ( $n=6$ for each tumour sample). When tumours reached approximately $5-10 \mathrm{~mm}$ in diameter, each tumour model was randomly divided into two groups $(n=3)$. In one group, $100 \mu$ l Lenti-hTERT-Fth1-3FLAG-Puro viral particles $\left(5 \times 10^{8} \mathrm{IU} / \mathrm{ml}\right)$ were intratumorally injected into the left-side tumour and nothing into the right-side tumour. In the other group, $100 \mu \mathrm{l}$ Lenti-CMV-Fth1-3FLAG-Puro viral particles $\left(5 \times 10^{8} \mathrm{IU} / \mathrm{ml}\right)$ were injected into the left-side tumour and $100 \mu \mathrm{l}$ PBS was injected into the right-side tumour.

\section{Magnetic resonance imaging}

Cells were grown in medium supplemented with FAC $(1 \mathrm{mM})$ for $48 \mathrm{~h}$ before MR imaging. After thorough washing with PBS to remove extracellular iron, dissociating with trypsin, and fixing in $4 \%$ PFA for $10 \mathrm{~min}$, the cells were suspended in $1 \mathrm{ml}$ PBS and transferred into 2-ml microcentrifuge tubes $\left(5 \times 10^{7}\right.$ cells/tube), which were precoated with $500 \mu l 1 \%$ agarose. After the cells had settled for 20-30 min and formed a loose pellet on the top of the agarose, MRI was performed with a 3.0-T MR unit with a knee coil (GE Signa HDx). T2*- 

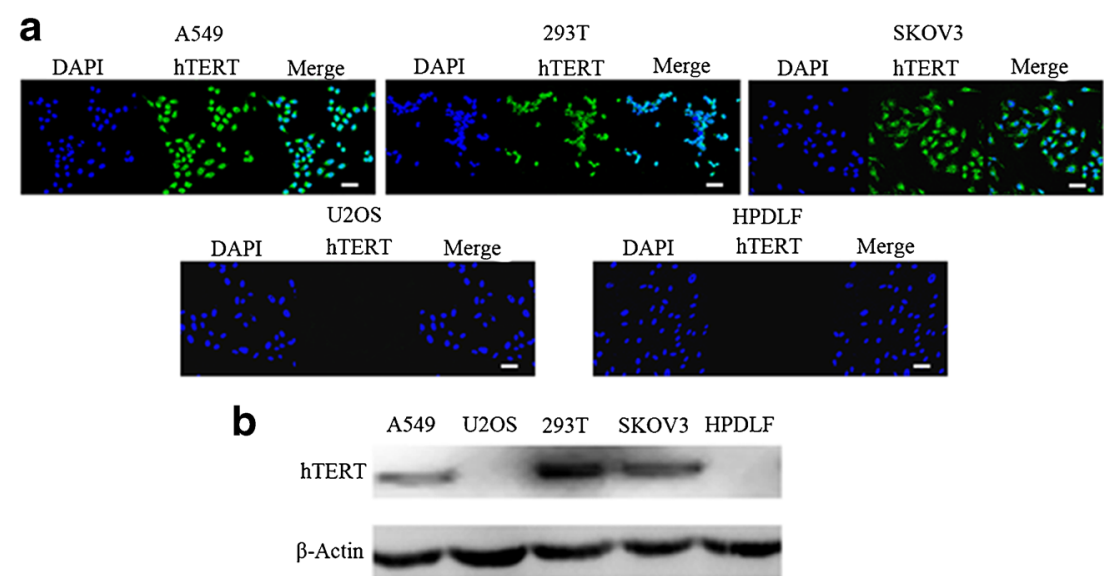

Fig. 1 Expression of hTERT protein in cell lines. a Immunofluorescence staining in cells with anti-hTERT antibody. In immunofluorescence images, hTERT was visible in A549, SKOV3 and 293T cells but not in U2OS or HPDLF cells. Green fluorescence represents hTERT proteins; blue fluorescence represents cell nuclei. Scale bar $50 \mu \mathrm{m}$. b Western blots for hTERT protein using anti-hTERT antibody ( $\beta$-actin was used as an internal control). hTERT protein was detected in A549, SKOV3 and 293T cells but not in U2OS or HPDLF cells weighted images were acquired using a GRE (gradient recalled echo) sequence with the following parameters: TR
$($ repetition time $)=400 \mathrm{~ms}$, TE $($ echo time $)=12 \mathrm{~ms}$, flip angle $=30^{\circ}$ and slice thickness $=1.0 \mathrm{~mm}$. In a second GRE
Fig. 2 Analysis of FLAG-tagged Fth expression in cells transfected with or without lentiviral vector. a Immunofluorescence staining of cells with anti-FLAG antibody. After transfection with LentihTERT-Fth1-3FLAG-Puro, FLAG-tagged protein was identified in immunofluorescence images of A549, SKOV3 and 293 T cells but not U2OS or HPDLF cells. Red fluorescence represents FLAG-tagged protein; blue fluorescence represents cell nuclei. Scale bar $50 \mu \mathrm{m}$. b ELISA for Fth protein in all cell types. In A549, SKOV3 and 293T cells, but not U2OS or HPDLF cells, transfected with Lenti-hTERTFth1-3FLAG-Puro, Fth protein level was significantly increased compared to the untransfected cells. Error bars indicate standard deviations of triplicate samples. ${ }^{*} p$ value was statistically significant $(p<0.05)$. hTERT Lenti-hTERT-Fth1-3FLAG-Purotransfected cells, $C M V$ LentiCMV-Fth1-3FLAG-Purotransfected cells, $W T$ wild-type cells a

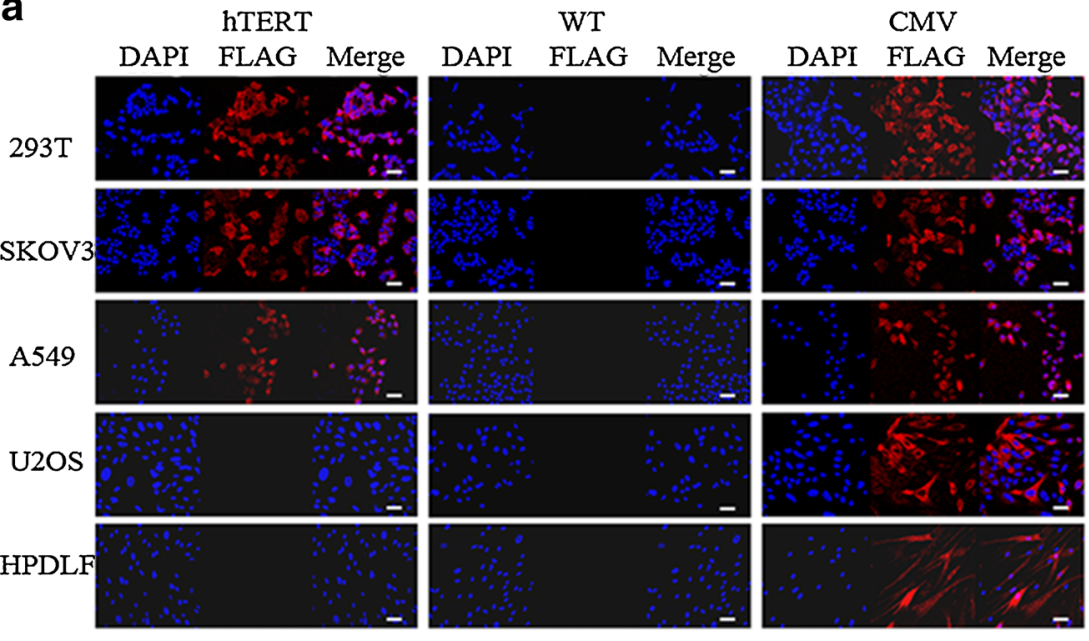

b

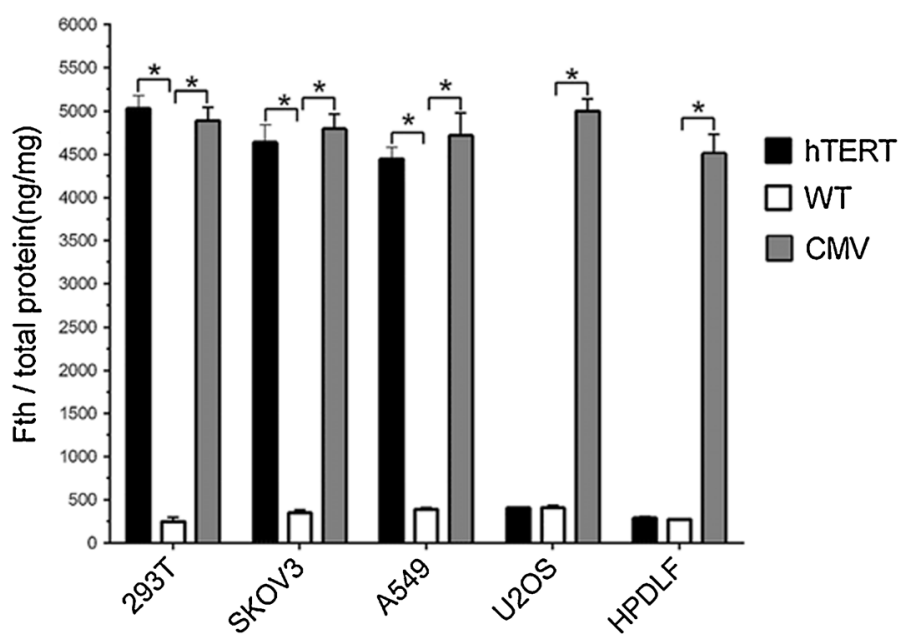


acquisition, R2* measurements were derived from eight images using the following parameters: $\mathrm{TR}=1000 \mathrm{~ms}, \mathrm{TE}=2.5-$ $27.0 \mathrm{~ms}$ ( 8 echoes) and slice thickness $=2.2 \mathrm{~mm}$.

Tumours were imaged 15 days after virus injection using a 1.5-T MR scanner (GE Signa HDx). Mice were anaesthetized with $2 \%$ sodium pentobarbital and placed in a wrist coil. T2weighted images of tumours were obtained using an FSE (fast spin echo) sequence (TR $=2000 \mathrm{~ms}, \mathrm{TE}=47.5 \mathrm{~ms}, \mathrm{FOV}$ (field of view) $=6 \times 6 \mathrm{~cm}$ and slice thickness $=2.0 \mathrm{~mm}$ ). T2 $*_{\text {- }}$ weighted images were obtained using a GRE sequence $(\mathrm{TR}=540 \mathrm{~ms}, \mathrm{TE}=10 \mathrm{~ms}, \mathrm{FOV}=6 \times 6 \mathrm{~cm}$ and slice thickness $=2.0 \mathrm{~mm}$ ).

\section{Immunohistochemistry and histology of tumour tissues}

After MR scanning, mice were decapitated, and tumours were removed and fixed with $4 \%$ PFA. Each tumour was embedded in OCT (optical cutting temperature) and sectioned using a cryostat microtome. Prepared frozen sections $(4 \mu \mathrm{m}$ in thickness) were used for immunofluorescence staining and Prussian blue iron staining to determine FLAG-tagged Fth expression and iron accumulation in tumour tissues, respectively, as described above.

\section{Statistical analysis}

Data are presented as the mean \pm SD. Significance tests were performed using one-way ANOVA (Graphpad Prism version 5.0). Differences were considered significant at $p$ values of less than 0.05 .

\section{Results}

\section{Expression of hTERT proteins in cell lines}

By performing immunofluorescence staining and western blot analysis, we confirmed the expression of hTERT proteins in A549, SKOV3 and 293T cell lines; whereas, no hTERT protein was detected in U2OS or HPDLF cell lines (Fig. 1). These results are consistent with previously published work [13-16]. As the expression of hTERT protein is good indicator of telomerase activity [2], A549, SKOV3 and 293T cell lines were considered telomerase-positive, while U2OS and HPDLF cell lines were considered telomerase-negative in this study.

\section{hTERT promoter drives Fth expression in telomerase-positive cells}

After transfection with Lenti-hTERT-Fth1-3FLAG-Puro, immunofluorescence staining showed that FLAG-tagged protein was present in telomerase-positive cells but absent in telomerase-negative cells. In contrast, FLAG-tagged protein was present in all five cell lines transfected with Lenti-CMVFth1-3FLAG-Puro and absent in all five untransfected cell lines (Fig. 2a). Analysis by ELISA showed that Fth protein content in Lenti-hTERT-Fth1-3FLAG-Puro-transfected telomerase-positive cells was significantly greater (4421-

a
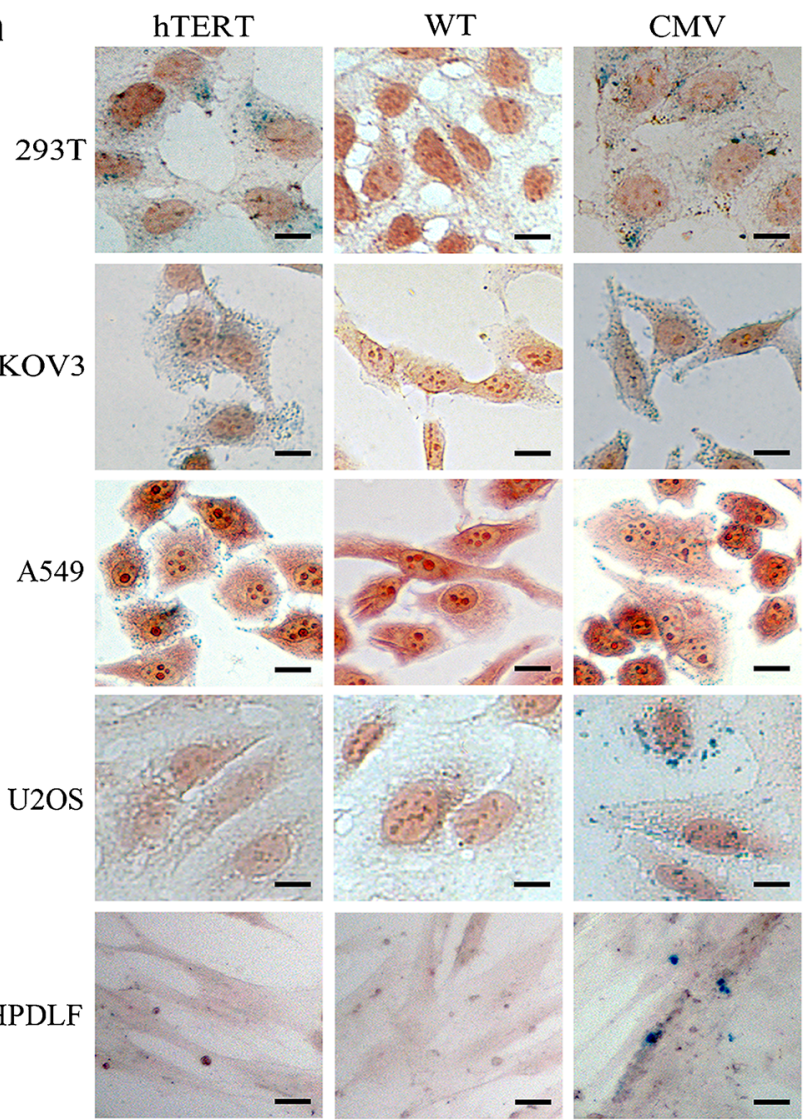

b

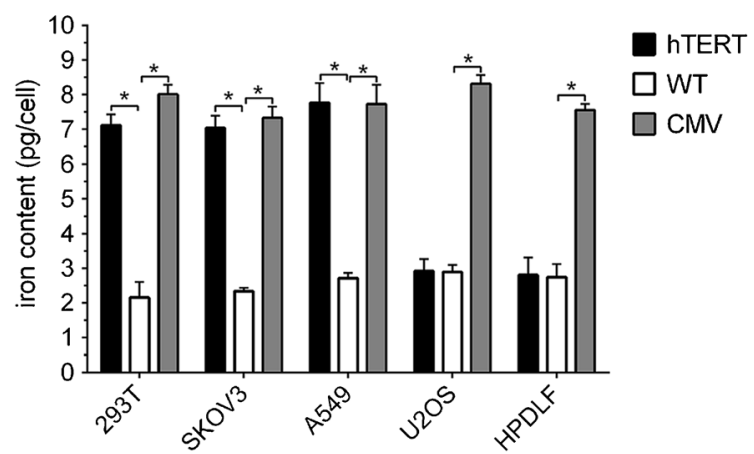

Fig. 3 Analysis of iron accumulation in cells treated with FAC (1 mM) for $48 \mathrm{~h}$. a Prussian blue staining of cells showed positive results in A549, SKOV3 and 293T cells, but not in U2OS or HPDLF cells, transfected with Lenti-hTERT-Fth1-3FLAG-Puro. Scale bar $5 \mu \mathrm{m}$. b Average iron content in each cell sample measured by an iron assay kit. A549, SKOV3 and 293 T cells, but not U2OS or HPDLF cells, transfected with LentihTERT-Fth1-3FLAG-Puro accumulated more iron than the untransfected cells. Error bars indicate standard deviations of triplicate samples. *p value was statistically significant $(p<0.05)$. hTERT Lenti-hTERT-Fth13FLAG-Puro-transfected cells, $C M V$ Lenti-CMV-Fth1-3FLAG-Purotransfected cells, WT wild-type cells 
$5039 \mathrm{ng} / \mathrm{mg}$ of total protein) than that in untransfected telomerase-positive cells (246-388 $\mathrm{ng} / \mathrm{mg}$ of total protein) $(p<0.05, n=3)$. In contrast, there was no remarkable difference between the Lenti-hTERT-Fth1-3FLAG-Purotransfected telomerase-negative cells and the untransfected telomerase-negative cells (270-409 $\mathrm{ng} / \mathrm{mg}$ of total protein) $(p>0.05, n=3)$ (Fig. 2b). By comparison, both telomerasepositive and telomerase-negative cells transfected with Lenti-CMV-Fth1-3FLAG-Puro contained greater Fth protein than their untransfected counterparts $(p<0.05, n=3)$ (Fig. 2b).

\section{More iron was accumulated in cells with Fth expression}

To verify the ability of Fth expression to augment iron storage, Prussian blue staining was performed. Blue granules of accumulated iron were detected in telomerase-positive cells but not in telomerase-negative cells transfected with Lenti-hTERTFth1-3FLAG-Puro. Moreover, blue granules were not observed in any untransfected cells (Fig. 3a). The iron content assay revealed that the iron content of Lenti-hTERT-Fth13FLAG-Puro-transfected telomerase-positive cells (7.033$7.766 \mathrm{pg} / \mathrm{cell})$ was significantly higher than that of untransfected telomerase-positive cells $(2.152-2.712 \mathrm{pg} / \mathrm{cell})$ $(p<0.05, n=3)$, but this difference was not observed in telomerase-negative cells (2.740-2.914 pg/cell) $(p>0.05$, $n=3$ ) (Fig. 3b). In contrast, all cells transfected with LentiCMV-Fth1-3FLAG-Puro showed blue granules and had significantly higher iron content than their untransfected counterparts $(p<0.05, n=3)$.

hTERT promoter-mediated Fth reporter gene expression induces specific MRI contrast in telomerase-positive cells in vitro

The T2*WI signal intensity of Lenti-hTERT-Fth1-3FLAGPuro-transfected telomerase-positive cells was lower than that of untransfected telomerase-positive cells, but this difference was not observed in telomerase-negative cells (Fig. 4a). In comparison, all of the Lenti-CMV-Fth1-3FLAG-Purotransfected cells showed signal loss compared to their untransfected counterparts (Fig. 4a). Furthermore, these signal changes were in agreement with the increase in cellular R2*. The R2* value of Lenti-hTERT-Fth1-3FLAG-Puro-transfected telomerase-positive cells $\left(110.8-134.9 \mathrm{~s}^{-1}\right)$ was higher than that of untransfected ones $\left(66.83-75.61 \mathrm{~s}^{-1}\right)(p<0.05)$, but this difference was not observed in telomerase-negative cells $\left(54.28-58.84 \mathrm{~s}^{-1}\right)(p>0.05)$ (Fig. 4b).

hTERT promoter-mediated Fth reporter gene expression induces specific MRI contrast in telomerase-positive tumours in vivo

A549 tumours showed hypointensity on T2*WI after injection with Lenti-hTERT-Fth1-3FLAG-Puro, while the U2OS tumours did not (Fig. 5). In contrast, both A549 and U2OS tumours showed hypointensity on $\mathrm{T} 2 * \mathrm{WI}$ after injection with Lenti-CMV-Fth1-3FLAG-Puro (Fig. 5). There was no apparent difference in signal intensity between the PBS-injected tumours and the WT (wild type) tumours (Fig. 5).

\section{Immunohistologic analysis of tumours}

Immunofluorescence staining of tumours with or without lentivirus injection was performed using anti-FLAG antibody. After injection with Lenti-hTERT-Fth1-3FLAG-Puro, FLAG-tagged Fth proteins were only expressed in A549 tumours (Fig. 6). In contrast, FLAG-tagged Fth proteins were detected in both tumour types after injection with Lenti-CMVFth1-3FLAG-Puro. Accordingly, notable positive Prussian blue staining was observed in Lenti-hTERT-Fth1-3FLAGPuro-injected A549 tumours and Lenti-CMV-Fth1-3FLAG- a

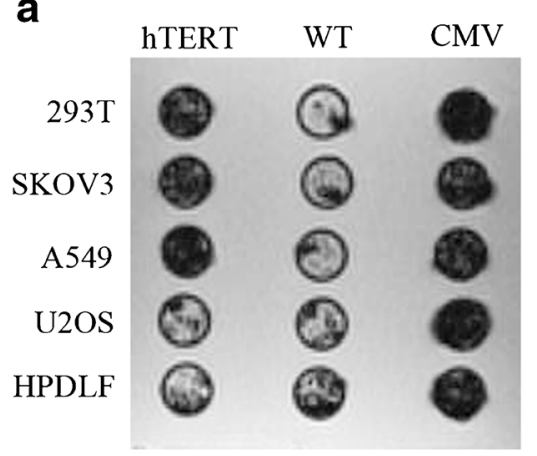

Fig. 4 In vitro MRI analysis of cells treated with FAC (1 mM) for $48 \mathrm{~h}$. a $\mathrm{T} 2 *$-weighted images of cells. Remarkable hypointensity was detected in A549, SKOV3 and 293T cells, but not U2OS or HPDLF cells, transfected with Lenti-hTERT-Fth1-3FLAG-Puro. b R2* values of cells were measured from R2* maps. Error bars indicate standard deviations of

b

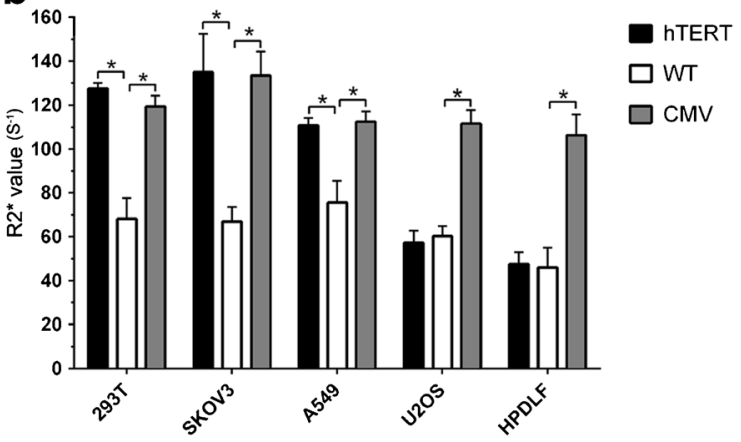

$\mathrm{R} 2 *$ within three regions of interest on the parametric map. ${ }^{*} p$ value was statistically significant $(p<0.05)$. hTERT Lenti-hTERT-Fth13FLAG-Puro-transfected cells, $C M V$ Lenti-CMV-Fth1-3FLAG-Purotransfected cells, $W T$ wild-type cells 


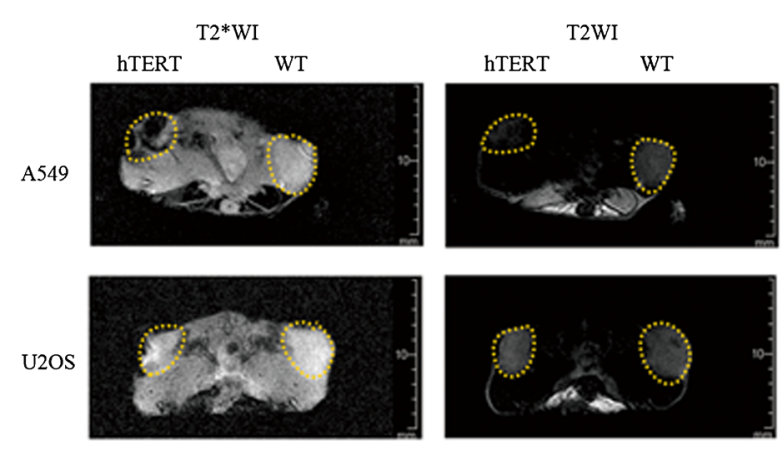

Fig. 5 In vivo MRI analysis of tumours injected with vectors. T2*weighted imaging of tumours shows remarkable hypointensity in A549 tumours, but not U2OS tumours, injected with Lenti-hTERT-Fth13FLAG-Puro and in A549 and U2OS tumours injected with Lenti-

Puro-injected A549 and U2OS tumours, but not in other tumours. These results were in line with those of the cell experiments described above.

\section{Discussion}

In recent years, great efforts have been devoted to designing molecular targeting reagents for cancer diagnosis. The research focus of many studies is on conjugating exogenous contrast agents with a specific ligand/antibody, so that the agents target the cells of interest and, thus, alter the MR signal intensity of those cells $[17,18]$. However, there are many limitations to this method, including the decay of receptorligand or antigen-antibody binding activity in vivo, the limited retention time of exogenous contrast agents in cells due to
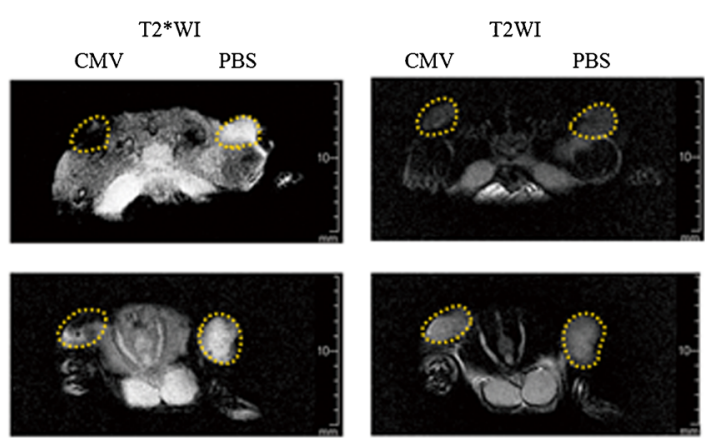

CMV-Fth1-3FLAG-Puro. No apparent signal loss in T2-weighted images of the tumours was observed. hTERT Lenti-hTERT-Fth13FLAG-Puro-injected tumours, $C M V$ Lenti-CMV-Fth1-3FLAG-Puroinjected tumours, $W T$ wild-type tumours, $P B S$ PBS-injected tumours

cellular division, and the background caused by nonspecific uptake of exogenous contrast agents by the reticuloendothelial system, which influences the visualization and analysis of cancer lesions.

Ferritin possesses a superparamagnetic moment and can shorten the T2 relaxation time of protons nearby [19]. The use of ferritin as a reporter gene to produce endogenous contrast can avoid some of the limitations of exogenous contrast agents. Cohen et al. first demonstrated that the introduced expression of ferritin heavy chain under tetracycline (TET) regulation resulted in an elevation of $\mathrm{R} 1$ and $\mathrm{R} 2$ relaxation rates in $\mathrm{C} 6$ cells, and they detected $\mathrm{C} 6$ tumours expressing ferritin heavy chain gene on T2WI [20]. Soon after, this team generated transgenic mice expressing the ferritin heavy chain under the control of the vascular endothelial cadherin promoter, and specifically visualized endothelial cells by MR
Fig. 6 Histological analyses of tumour sections. a Immunofluorescence staining of FLAG protein in tumours with anti-FLAG antibody. Red fluorescence represents FLAGtagged proteins; blue fluorescence represents cell nuclei. Scale bar $50 \mu \mathrm{m}$. b Prussian blue staining of iron deposits in tumour tissues. Scale bar $10 \mu \mathrm{m}$. hTERT LentihTERT-Fth1-3FLAG-Puroinjected tumours, $C M V$ LentiCMV-Fth1-3FLAG-Puroinjected tumours, $W T$ wild-type tumours

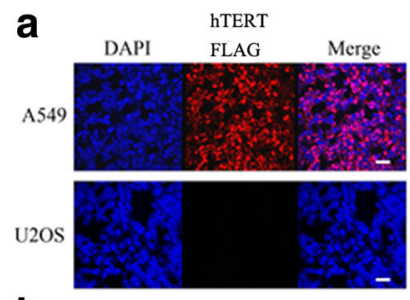

b

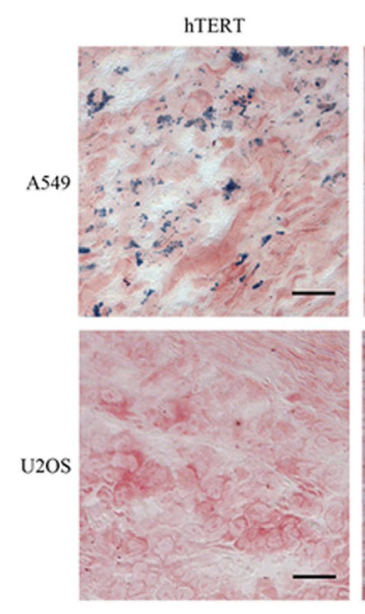

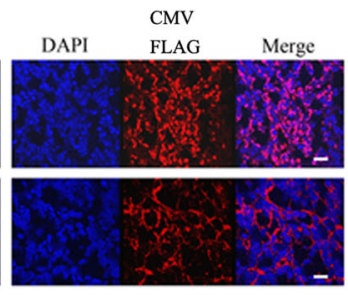

CMV

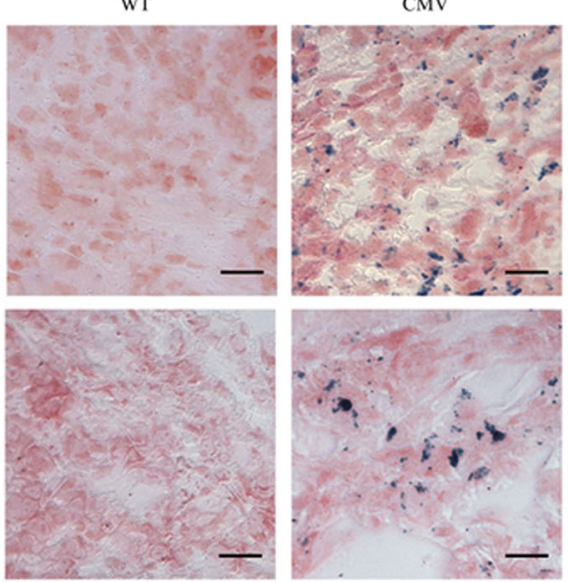


imaging [21]. To date, a variety of promoters have been explored and used to regulate target gene expression, e.g. promoters selected on the basis of their potential to target cancer, such as the prostate-specific antigen promoter [22], $\alpha$ fetoprotein promoter [23] and the prolactin promoter [24].

In the present study, we used the hTERT promoter to drive ferritin heavy chain expression, since it is functional in the majority of cancer cells regardless of their histological types, but is silent in somatic or benign cells. We constructed a FLAG-tagged ferritin heavy chain reporter gene expression lentiviral vector Lenti-hTERT-Fth1-3FLAG-Puro and showed that FLAG-tagged Fth was specifically expressed in telomerase-positive cells both in vitro and in vivo. In addition, Prussian blue staining for iron was observed in Fth-expressing cells and tumours. With the increase in ferritin protein and iron accumulation, the R2* value of cells was significantly increased and signal loss was observed on $\mathrm{T} 2 *$-weighted images. However, we found that the results obtained above did not correspond very well with each other, possibly because of the contribution of many factors to R $2 *$, such as the mode of nucleation of iron, the aggregation of ferritin in lysosomes [25], different levels of promoter activity and different levels of ferritin expression in different cell lines. As shown in Fig. 5, signal loss was observed on GRE T2*-weighted images of tumours with Fth reporter gene expression but not on FSE T2-weighted images, suggesting that a GRE sequence is likely more sensitive to iron than FSE sequence [26].

Moreover, the Fth-expressing tumours also showed heterogeneous signal intensity on $\mathrm{T} 2 *$-weighted images. There are several possible explanations for this finding: (1) the uneven distribution of iron particles in the tumour tissues; (2) the manner of lentivirus injection, which may cause cells near the injection site to be transfected with more vector particles than those distant from the injection site, resulting in a higher ferritin expression level in the cells near the injection site; and (3) the maldistribution of telomerase-positive cells in the lesions, as the hTERT promoter can only drive ferritin expression in them.

In summary, we used an MR molecular imaging technology to specifically detect cancer cells both in vitro and in vivo. We demonstrate for the first time that MR reporter imaging mediated by the hTERT promoter could be a novel and broadspectrum imaging tool for cancer research and diagnosis.

\footnotetext{
Acknowledgments The study was supported by the National Science Foundation of China (no.81471635) and the Science and Technology Commission of ChongQing City, China (no.cstc2013jjB10010). The scientific guarantor of this publication is Zhang Dong. The authors of this manuscript declare no relationships with any companies whose products or services may be related to the subject matter of the article. No complex statistical methods were necessary for this paper. Institutional review board approval was obtained. Approval from the Institutional Animal Care and Use Committee of the Third Military Medical University was obtained.
}

Open Access This article is distributed under the terms of the Creative Commons Attribution-NonCommercial 4.0 International License (http://creativecommons.org/licenses/by-nc/4.0/), which permits any noncommercial use, distribution, and reproduction in any medium, provided you give appropriate credit to the original author(s) and the source, provide a link to the Creative Commons license, and indicate if changes were made.

\section{References}

1. Dhaene K, Van Marck E, Parwaresch R (2000) Telomeres, telomerase and cancer: an up-date. Virchows Arch 437:1-16

2. Saldanha SN, Andrews LG, Tollefsbol TO (2003) Analysis of telomerase activity and detection of its catalytic subunit, hTERT. Anal Biochem 315:1-21

3. Cong YS, Wen J, Bacchetti S (1999) The human telomerase catalytic subunit hTERT: organization of the gene and characterization of the promoter. Hum Mol Genet 8:137-142

4. Kyo S, Takakura M, Fujiwara T, Inoue M (2008) Understanding and exploiting hTERT promoter regulation for diagnosis and treatment of human cancers. Cancer Sci 99:1528-1538

5. Gilad AA, Winnard PT Jr, van Zijl PC, Bulte JW (2007) Developing MR reporter genes: promises and pitfalls. NMR Biomed 20:275-290

6. Massoud TF, Gambhir SS (2003) Molecular imaging in living subjects: seeing fundamental biological processes in a new light. Genes Dev 17:545-580

7. Groot-Wassink T, Aboagye EO, Wang Y, Lemoine NR, Keith WN, Vassaux G (2004) Noninvasive imaging of the transcriptional activities of human telomerase promoter fragments in mice. Cancer Res 64:4906-4911

8. Yu ST, Yang YB, Liang GP et al (2010) An optimized telomerasespecific lentivirus for optical imaging of tumors. Cancer Res 70: 2585-2594

9. Lee SW, Lee SH, Biswal S (2012) Magnetic resonance reporter gene imaging. Theranostics 2:403-412

10. Hasegawa S, Furukawa T, Saga T (2010) Molecular MR imaging of cancer gene therapy: ferritin transgene reporter takes the stage. Magn Reson Med Sci 9:37-47

11. Davis HE, Morgan JR, Yarmush ML (2002) Polybrene increases retrovirus gene transfer efficiency by enhancing receptorindependent virus adsorption on target cell membranes. Biophys Chem 97:159-172

12. Kim HS, Cho HR, Choi SH, Woo JS, Moon WK (2010) In vivo imaging of tumor transduced with bimodal lentiviral vector encoding human ferritin and green fluorescent protein on a $1.5 \mathrm{~T}$ clinical magnetic resonance scanner. Cancer Res 70:7315-7324

13. Braunstein I, Cohen-Barak O, Shachaf C et al (2001) Human telomerase reverse transcriptase promoter regulation in normal and malignant human ovarian epithelial cells. Cancer Res 61:5529-5536

14. Fujii S, Maeda H, Wada N, Kano Y, Akamine A (2006) Establishing and characterizing human periodontal ligament fibroblasts immortalized by SV40T-antigen and hTERT gene transfer. Cell Tissue Res 324:117-125

15. Irving J, Wang Z, Powell S et al (2004) Conditionally replicative adenovirus driven by the human telomerase promoter provides broad-spectrum antitumor activity without liver toxicity. Cancer Gene Ther 11:174-185

16. Padmanabhan P, Otero J, Ray P et al (2006) Visualization of telomerase reverse transcriptase (hTERT) promoter activity using a trimodality fusion reporter construct. J Nucl Med 47:270-277

17. Ren J, Wang F, Wei G et al (2012) MRl of prostate cancer antigen expression for diagnosis and lmmunotherapy. PLoS ONE 7:e38350 
18. Yang HM, Park CW, Woo MA et al (2010) HER2/neu antibody conjugated poly(amino acid)-coated iron oxide nanoparticles for breast cancer MR imaging. Biomacromolecules 11:2866-2872

19. Brooks RA, Vymazal J, Goldfarb RB, Bulte JW, Aisen P (1998) Relaxometry and magnetometry of ferritin. Magn Reson Med 40: 227-235

20. Cohen B, Dafni H, Meir G, Harmelin A, Neeman M (2005) Ferritin as an endogenous MRI reporter for noninvasive imaging of gene expression in C6 glioma tumors. Neoplasia 7: 109-117

21. Cohen B, Ziv K, Plaks V et al (2007) MRI detection of transcriptional regulation of gene expression in transgenic mice. Nat Med 13:498-503

22. Willhauck MJ, Sharif Samani BR, Gildehaus FJ et al (2007) Application of 188rhenium as an alternative radionuclide for treatment of prostate cancer after tumor-specific sodium iodide symporter gene expression. J Clin Endocrinol Metab 92:44514458

23. Kim KI, Lee YJ, Lee TS et al (2013) In vitro radionuclide therapy and in vivo scintigraphic imaging of alpha-fetoprotein-producing hepatocellular carcinoma by targeted sodium iodide symporter gene expression. Nucl Med Mol Imaging 47:1-8

24. Stirland JA, Seymour ZC, Windeatt S et al (2003) Real-time imaging of gene promoter activity using an adenoviral reporter construct demonstrates transcriptional dynamics in normal anterior pituitary cells. J Endocrinol 178:61-69

25. Cohen B, Ziv K, Plaks V, Harmelin A, Neeman M (2009) Ferritin nanoparticles as magnetic resonance reporter gene. Wiley Interdiscip Rev Nanomed Nanobiotechnol 1:181-188

26. Juchems MS, Cario H, Schmid M, Wunderlich AP (2012) Liver iron content determined by MRI: spin-echo vs. gradient-echo. Röfo $184: 427-431$ 